\title{
Extended foot-ankle musculoskeletal models for application in movement analysis
}

\author{
Tiago M. Malaquias ${ }^{1 *}$, Carolina Silveira ${ }^{2}$, Wouter Aerts ${ }^{1}$, Friedl De Groote ${ }^{3}$, Greta \\ Dereymaeker $^{1}$, Jos Vander Sloten ${ }^{1}$, Ilse Jonkers ${ }^{3}$ \\ 1 Department of Mechanical Engineering, Biomechanics Section, KU Leuven, Belgium \\ 2 Department of Physics, University of Coimbra, Portugal \\ 3 Department of Kinesiology, Human Movement Biomechanics Research Group, KU Leuven, \\ Belgium \\ *melo.malaquias@kuleuven.be
}

\begin{abstract}
Multibody simulations of human motion require representative models of the anatomical structures. A model that captures the complexity of the foot is still lacking. In the present work, two detailed 3D multibody foot-ankle models generated based on CT scans using a semi-automatic tool are described. The proposed models consists of five rigid segments (talus, calcaneus, midfoot, forefoot and toes), connected by five joints (ankle, subtalar, midtarsal, tarsometatarsal and metatarsophalangeal), one with 15DOF and the other with 8DOF. The calculated kinematics of both models were evaluated using gait trials and compared against literature, both presenting realistic results. An inverse dynamic analysis was performed for the $8 \mathrm{DOF}$ model, again presenting feasible dynamic results.
\end{abstract}

\section{Keywords}

Movement analysis, Foot, Three-dimensional computer modeling, Multibody simulation, CT-Scan

\section{Acknowledgments}

This work was funded by the Berghmans - Dereymaeker research chair on Foot and Ankle Biomechanics and the Aladyn Project (IWT, Flanders). 


\section{Introduction}

The foot is a highly complex structure. Nevertheless, musculoskeletal models commonly describe it by simplified kinematic and dynamic models with three or fewer segments (Anderson \& Pandy 2001; Bruening et al. 2012). Being an intricate structure, a small number of segments is generally insufficient to capture the detail of human foot motion and joint loading (Neptune et al. 2000).

In the present work, two extended multibody foot-ankle models that capture important foot movements at the midtarsal and tarsometatarsal (mentioned in Hicks 1953) were developed. A tool to construct 3D multibody foot-ankle models based on CT scans for use in OpenSim (Delp

et al. 2007) was created. Both models consisted of five rigid segments (talus, calcaneus, midfoot, forefoot and toes) with five anatomical joints (ankle, subtalar, midtarsal, tarsometatarsal and metatarsophalangeal) with fifteen and eight degrees of freedom (DOF) respectively. The first model has 3DOF at each joint, the DOF of the second model were based on the work of Hicks (1953). In addition, the geometry of the intrinsic and extrinsic muscles were included as well as the major ligaments. Both models were validated by comparing inverse kinematics for gait with joint kinematics published in literature (Lundgren et al. 2008). Furthermore, the 8DOF model was used to evaluate inverse dynamics and ligament strain during gait.

\section{Methods}

The following workflow describes the methodology applied to develop both models.

\section{Surfaces and volume meshes generation}

CT scan foot data from one healthy subject was segmented using Mimics ${ }^{\circledR}$ Innovation Suite (Materialise, Leuven, Belgium). The same software was used to create the surfaces and volume meshes of the bones and the soft tissues. The soft tissue meshes were only used for the computation of the segments' mass and inertia tensor. The bone segments were generated 
through the union of the corresponding bones: midfoot (navicular, cuboid and cuneiforms), forefoot (metatarsals) and toes (phalanxes). Each segment was exported as a surface mesh (triangles) and as a volume mesh (tetrahedrons) separately. The soft tissue was divided in four different regions (calcaneus, midfoot, forefoot and toes) using cutting planes. Each plane was defined using three anatomical landmarks:

- Midtarsal Plane - Central point of the lateral side of the cuboid at the level of the calcaneal-cuboid joint line, central point of the medial side and central point of the dorsal side of the navicular bone at the level of the talus-navicular joint line;

- Tarsometatarsal Plane - Central point of the medial side of the $1^{\text {st }}$ metatarsal bone at the level of the metatarsal-cuneiform joint line, central point of the lateral side of the $5^{\text {th }}$ metatarsal bone at the level of metatarsal-cuboid joint line and the highest central point of the dorsal side of the $2^{\text {nd }}$ metatarsal bone;

- Metatarsophalangeal Plane - Lowest central point at the plantar side of the $1^{\text {st }}$ proximal phalanx, highest central point of the $1^{\text {st }}$ proximal phalanx and central point of the lateral side of the $5^{\text {th }}$ proximal phalanx.

\section{Computation of the local coordinate systems}

An Euclidean transform in 3D space, based on single value decomposition (Besl \& McKay 1992), was used to transform the foot segment meshes from the CT scan coordinate system to OpenSim based on four corresponding points in both feet (CT scan and OpenSim - Model Gait 2392). A reference frame with origin at the joint center was computed for each segment based on reproducible anatomical landmarks (Table 1), selected on the bones mesh using Mimics® Innovation Suite. 


\section{Anthropometric properties computation}

The segments properties (volume, mass and center of mass) were computed using the volume mesh information and the CT scan's greyscale of each element. The inertia tensor was computed based on the density information using explicit formulas for the moments of inertia of 3D tetrahedrons as polynomials of their vertex coordinates (Tonon 2004).

Mimics® Innovation Suite allowed to assign a density value $(\rho)$ to each volume mesh element $(n)$ based on a linear relation between the CT scan's greyscale (Hounsfield units - $H U$ ) and the correspondent density value (Equation 1). In total, 10 different densities were assigned to each segment $(i)$, with each density value correlating to an equally spaced set of HU measures.

$$
\rho_{n, i}=b_{i}+a_{i} \cdot H U_{n}
$$

The line's slope (a) and the $y$-intersect $(b)$ for each segment resulted from the following system of equations:

$$
\left\{\begin{array}{c}
\rho_{\text {Trabecular }}=b_{i}+a_{i} \cdot H U_{\min _{i}} \\
\rho_{\text {Cortical }}=b_{i}+a_{i} \cdot H U_{\max _{i}}
\end{array}\right.
$$

where $H U_{\min _{i}}$ and $H U_{\max _{i}}$ are the minimum and maximum greyscale value for each segment, and represent the trabecular and cortical bone. The density values used in Equation 2 for the trabecular and cortical bone were $0.05 \times 10^{3} \mathrm{~kg} / \mathrm{m}^{3}$ and $1.75 \times 10^{3} \mathrm{~kg} / \mathrm{m}^{3}$ respectively, based on femur bone density values, which were assumed to have similar densities to foot bones (Bitsakos et al. 2005). For the soft tissue a uniform density $\left(\rho_{S T}=1.0158 \times 10^{3} \mathrm{~kg} / \mathrm{m}^{3}\right)$ was used by averaging muscle (Ward \& Lieber 2005) and fat density (Farvid et al. 2005).

A lower limit of $0.001 \mathrm{~kg} \cdot \mathrm{m}^{2}$ was established for the principal direction of the inertia tensor to avoid problems during the dynamic simulation. 


\section{Model creation}

Using all the information previously described both models were automatically generated using the Matlab-OpenSim interface (see Figure 1).

The 15DOF model has 3DOF at each joint (Plantarflexion/Dorsiflexion, Abduction/Adduction, and Supination/Pronation). The 8DOF model is based on the work of Hicks (1953) and has

- one DOF at the ankle joint - plantarflexion/dorsiflexion;

- one DOF at the subtalar joint - defined by an oblique axis, combining eversion-abductionextension/inversion-adduction-flexion;

- two DOF at the midtarsal joint - defined by two axes, an oblique and an anterior-posterior axis. Both allowing a combination of eversion-abduction-extension/inversion-adductionflexion.

- two DOF at the tarsometatarsal joint - defined by two axes, the $1^{\text {st }}$ ray axis and the 5th ray axis, both allowing flexion-eversion/extension-eversion;

- two DOF at the metatarsophalangeal joint - plantarflexion/dorsiflexion and abduction/adduction.

36 ligaments that span at least two of the defined segments were included in our model (Table 2). The implementation of these structures required a geometrical path, described by an anatomical based point set (origin, insertion and optional via points) (Kelikian \& Sarrafian 1983).

\section{Marker set protocol and Acquisition}

Experimental data collected in five healthy subjects, four trials per subject and per foot, walking barefoot were used evaluate the foot model. Motion capture data was collected using ten infrared Vicon cameras (Vicon, Oxford Metrics, UK) to track the motion of 65 skin-mounted markers (18 
positioned on each foot) at a sampling rate of $200 \mathrm{~Hz}$ (Duerinck et al. 2014). Synchronized ground reaction forces (GRF) and plantar pressure data were collected. Consistency of the normal force computed from the pressure data with the vertical component of the GRF was verified and confirmed in all trials. Force data was collected at $1000 \mathrm{~Hz}$ using two AMTI force plates (Advanced Medical Technology, Watertown, Massachusetts) embedded in the walkway. Plantar pressure plates (RSscan International NV) were positioned on top of these force plates and recorded data at a sampling rate of $500 \mathrm{~Hz}$. The study was approved by the local ethical committee and all participants provided written informed consent.

\section{Decomposition of ground reaction forces}

The measured GRF were decomposed into four components applied to the calcaneus, midfoot, forefoot and toes respectively for use in inverse dynamics simulation. At each time frame, the GRF were decomposed proportional to the measured plantar pressure under each foot segment using a manually defined mask based on the maximal plantar pressure map over all time steps.

\section{Validation}

A total of 40 trials was used. The model was scaled in Opensim for each subject using a static measurement. Joint kinematics were estimated for both models (8DOF and 15DOF) using a Kalman smoothing algorithm for inverse kinematics (De Groote et al. 2008). 7 trials for the 15DOF model and 4 for the 8DOF model were left out because the outputted kinematic solution

presented a physiologically unrealistic movement due to an inaccurate marker data. Based on the joint kinematics, the relative orientation of the segments was computed.

Using the computed kinematics the elongation of the ligaments was calculated as a percentage of their resting length during the stance phase of gait. The joint moments of the 8DOF model were calculated using an inverse dynamics analysis 


\section{Results}

The mean relative motion between segments, mean joint moments and mean ligament elongation, and their correspondent standard deviation (SD) are displayed in Figure 2. The kinematic results are compared against the in-vivo foot kinematics data of Lundgren et al. (2008). In this work they used bone anchored external markers to describe the kinematics, a more direct measurement of skeletal kinematics than skin mounted markers which are prone to soft tissue artefacts.

The kinematics computed with the 15DOF model presents more inter-subject variability when comparing to the $8 \mathrm{DOF}$ model (i.e. maximal $S D_{\text {Tibia-Talus }}^{15 \text { OOF }}=8.3^{\circ}$ against maximal $S D_{\text {Tibia-Talus }}^{8 D O F}=6 \cdot 3^{\circ}$. The mean kinematics comparison between both models and literature, using the root mean square (RMS), are generally consistent (i.e. $R M S_{\text {Tibia-Talus }}^{8 D O F}=2.0^{\circ}$ and $R M S_{\text {Calcaneus-Midfoot }}^{8 D O F}=1.7^{\circ}, R M S_{\text {Tibia-Talus }}^{15 D O F}=2.0^{\circ}$ and $\left.R M S_{\text {Calcaneus-Midfoot }}^{15 \text { DOF }}=2.7^{\circ},\right)$. The largest differences are observed in the motion between the talus and midfoot $\left(R M S_{\text {Talus }}^{8 D O F}\right.$ Midfoot $=8.8^{\circ}$ and $R M S_{\text {Talus-Midfoot }}^{15 D O F}=9.3^{\circ}$.

Since kinematics computed with the 8DOF model was less variable (smaller SD) and to some extent more in accordance with literature (smaller RMS) than kinematics computed with the 15DOF model, the dynamics analysis was only performed for the 8DOF model. The SD of the joint torques (Figure 2 - B) is small (i.e. maximal $S D_{\text {Subtalar }}^{8 D O F}=0.13 \mathrm{Nm} / \mathrm{kg}$ and maximal $S D_{\text {Tarsometatarsal } 5 \text { th Ray }}^{8 D O F}=0.087 \mathrm{Nm} / \mathrm{kg}$ ), indicating a high inter-subject consistency. Furthermore, the definition of the ligaments' geometry allowed the assessment of their elongation during gait compared to their length in anatomical position, presenting an average maximum elongation for the ankle ligaments of $5.3 \%$. 


\section{Discussion}

The 8DOF model resulted in kinematics that better resemble literature than the 15DOF model. The 15DOF model, due to the three rotational DOF in each joint, has more freedom to solve the inverse kinematics problem and is thus more prone to measurement errors, which might explain the higher standard deviations. The main kinematic differences, when comparing to Lundgren et al. (2008), are observed in the movements between talus and midfoot. However, this difference might be explained by the fact that the model evaluates the movement between segments (talus and midfoot) whereas Lundgren et al. measured the movement between talus and navicular, which might move with respect to the other bones in the midfoot. Since the work of Lundgren et al. (2008) did not include bone pins in the phalanges, it was not possible to compare the metatarsophalangeal joint kinematics. Likewise, no reference data for the relative movement of the midfoot-forefoot was available.

To date, only a limited number of foot models provide dynamic joint information for other joints than the ankle, making the comparison of our results challenging. Nevertheless, the presented results are consistent with the available literature for the joint moments at the ankle, subtalar, midtarsal, and metatarsophalangeal joints (Scott \& Winter 1993; Bruening et al. 2012; Malaquias et al. 2015).

The ankle ligaments average maximum elongation showed a physiological acceptable value, since ligament rupture occurs above $8 \%$ (Nordin \& Frankel 2001). However, for the smaller foot ligaments, further refinement is needed.

\section{Conclusions}

A simple, reproducible and efficient semi-automatic tool to compute multi-segment foot models based on CT scans was developed, allowing a more detailed representation of the ankle-foot 
complex with 8DOF and 15DOF. A total of 36 ligaments were included in both models to allow evaluating their elongation. The 8DOF model-based inverse kinematic and inverse dynamic analysis of gait resulted in realistic kinematics and dynamics. Due to the more accurate representation of the degrees of freedom in the foot, this model has the potential to offer new insights in pathological foot kinematics and dynamics.

Future research should concentrate on quantifying the specific dynamic parameters of the intrinsic muscles and ligaments (e.g. ligament resting length, stiffness, muscle's optimal fiber length and tendon slack length), allowing the study of muscle and ligament forces.

\section{Supplementary Material}

The model is made available in: simtk.org/home/kul_footmodel.

\section{References}

Anderson FC, Pandy MG. 2001. Dynamic Optimization of Human Walking. Journal of Biomechanical Engineering. 123:381-390.

Besl P, McKay N. 1992. A Method for Registration of 3-D Shapes. IEEE Transactions on Pattern Analysis and Machine Intelligence. 14:239-256.

Bitsakos C, Kerner J, Fisher I, Amis AA. 2005. The effect of muscle loading on the simulation of bone remodelling in the proximal femur. Journal of Biomechanics. 38:133-139.

Bruening DA, Cooney KM, Buczek FL. 2012. Analysis of a kinetic multi-segment foot model part II: kinetics and clinical implications. Gait \& Posture. 35:535-40.

Delp SL, Anderson FC, Arnold AS, Loan P, Habib A, John CT, Guendelman E, Thelen DG. 2007. OpenSim: Open-source software to create and analyze dynamic simulations of movement. IEEE Transactions on Biomedical Engineering. 54:1940-1950.

Duerinck S, Hagman F, Jonkers I, Roy P Van, Vaes P. 2014. Forefoot deformation during stance : Does the forefoot collapse during loading? Gait \& Posture. 39:40-47. 
Farvid MS, Ng TWK, Chan DC, Barrett PHR, Watts GF. 2005. Association of adiponectin and resistin with adipose tissue compartments, insulin resistance and dyslipidaemia. Diabetes, Obesity and Metabolism. 7:406-413.

De Groote F, De Laet T, Jonkers I, De Schutter J. 2008. Kalman smoothing improves the estimation of joint kinematics and kinetics in marker-based human gait analysis. Journal of Biomechanics. 41:3390-3398.

Hicks JH. 1953. The Mechanics of the Foot I-The Joints. Journal of Anatomy. 87:345-357.

Kelikian AS, Sarrafian SK. 1983. Sarrafian's Anatomy of the Foot and Ankle: Descriptive, Topographic, Functional. Philadelphia: Lippincott Williams \& Wilkins.

Lundgren P, Nester C, Liu A, Arndt A, Jones R, Stacoff A, Wolf P, Lundberg A. 2008. Invasive in vivo measurement of rear- , mid- and forefoot motion during walking. Gait \& Posture. 28:93100.

Malaquias TM, Gonçalves SB, da Silva MT. 2015. A Three-Dimensional Multibody Model of the Human Ankle-Foot Complex. In: Flores P, Viadero F, editors. New Trends in Mechanism and Machine Science SE - 47. Vol. 24. Cham: Springer International Publishing; p. 445-453.

Neptune RR, Wright IC, Van Den Bogert AJ. 2000. A method for numerical simulation of single limb ground contact events: Application to Heel-Toe Running. Computer Methods in Biomechanics and Biomedical Engineering. 3:321-334.

Nordin M, Frankel VH. 2001. Basic Biomechanics of the Musculoskeletal System. 3rd ed. Philadelphia: Lippincott Williams \& Wilkins.

Scott SH, Winter DA. 1993. Biomechanical model of the human foot: kinematics and kinetics during the stance phase of walking. Journal of Biomechanics. 26:1091-1104.

Tonon F. 2004. Explicit Exact Formulas for the 3-D Tethraedron Inertia Tensor in Terms of its Vertex Coordinates. Journal of Mathematics and Statistics. 1:8-11.

Ward SR, Lieber RL. 2005. Density and hydration of fresh and fixed human skeletal muscle. Journal of Biomechanics. 38:2317-2320. 
Table 1 - Anatomical landmarks, selected on the bones mesh, used to compute the joint axes and segments origin.

\begin{tabular}{lc}
\hline Bones & Landmarks \\
\hline
\end{tabular}

Talus

Supero-medial aspect of the neck of the Talus

Tip of the Lateral Process

Center of the Ankle joint

(Medium Point)

Tip of the Medial Malleolus

Tip of the Lateral Malleolus

Calcaneus

Sustentaculum Tali

Postero-lateral 'corner' of the Calcaneus

Center of the Calcaneus/Cuboid joint

Center of the Subtalar joint

(Center of the sphere defined by four points)

Center of the posterior articular surface

Center of the middle articular surface

Center of the anterior articular surface

Tip of the trochlear process

Midfoot

Supero medial aspect of the head of the Talus

Tip of the Lateral process of the Calcaneus

Center of the midtarsal joint

Medial medium point of the Talo-Navicular joint

(Medium Point)

Lateral medium point of the Calcaneo-Cuboid joint

Forefoot

Mid-dorsum of the foot over the base of the $3^{\text {rd }}$ metatarsal

Tuberosity of the Navicular

Center of the Tarsometatarsal joint

Lateral tuberosity of the $5^{\text {th }}$ Metatarsal

(Medium Point)

Medial medium point of the Cuneiform- $1^{\text {st }}$ Metatarsal articular surface

Toes

Tip of the $1^{\text {st }}$ Distal Phalanx

Center of the Metatarsophalangeal joint

Medial medium point of the $1^{\text {st }}$ Metatarsal- $1^{\text {st }}$ proximal Phalanx articular

(Medium Point)

Lateral medium point of the $5^{\text {th }}$ Metatarsal $-5^{\text {th }}$ proximal Phalanx articular 
Table 2 - Ligaments geometrical path description. The Resting Length is presented for the generic model, before scaling.

\begin{tabular}{|c|c|c|c|c|}
\hline Ligament & Origin & Via Points & Insertion & Resting Length (m) \\
\hline Tibio-Talar Posterior & $\overline{\text { Tibia }}$ & - & $\overline{\text { Talus }}$ & 0.01982 \\
\hline Tibio-Talar Anterior & Tibia & - & Talus & 0.02534 \\
\hline Tibio-Calcaneal & Tibia & - & Calcaneus & 0.03015 \\
\hline Tibio-Navicular & Tibia & - & Navicular & 0.04080 \\
\hline Talo-Fibular Posterior & Tibia & - & Talus & 0.03009 \\
\hline Talo-Fibular Anterior & Tibia & - & Talus & 0.03016 \\
\hline Calcaneo-Fibular & Tibia & - & Calcaneus & 0.03571 \\
\hline Plantar Fascia & Calcaneus & $1^{\text {st }}$ Metatarsal & $1^{\text {st }}$ Prox. Phalange & 0.14290 \\
\hline Plantar Fascia & Calcaneus & $2^{\text {nd }}$ Metatarsal & $2^{\text {nd }}$ Prox. Phalange & 0.14404 \\
\hline Plantar Fascia & Calcaneus & $3^{\text {rd }}$ Metatarsal & $3^{\text {rd }}$ Prox. Phalange & 0.14335 \\
\hline Plantar Fascia & Calcaneus & $4^{\text {th }}$ Metatarsal & $4^{\text {th }}$ Prox. Phalange & 0.13612 \\
\hline Plantar Fascia & Calcaneus & $5^{\text {th }}$ Metatarsal & $5^{\text {th }}$ Prox. Phalange & 0.12815 \\
\hline Longitudinal Plantar & Calcaneus & Cuboid & $2^{\text {nd }}$ Metatarsal & 0.08037 \\
\hline Longitudinal Plantar & Calcaneus & Cuboid & $3^{\text {rd }}$ Metatarsal & 0.07665 \\
\hline Longitudinal Plantar & Calcaneus & Cuboid & $4^{\text {th }}$ Metatarsal & 0.07153 \\
\hline Longitudinal Plantar & Calcaneus & Cuboid & $5^{\text {th }}$ Metatarsal & 0.06389 \\
\hline Calcaneo-Navicular Plantar & Calcaneus & - & Navicular & 0.01968 \\
\hline Calcaneo-Navicular Plantar & Calcaneus & - & Navicular & 0.01214 \\
\hline Calcaneo-Navicular Plantar & Calcaneus & - & Navicular & 0.01041 \\
\hline Calcaneo-Cuboid Plantar & Calcaneus & - & Cuboid & 0.01767 \\
\hline Calcaneo-Cuboid Plantar & Calcaneus & - & Cuboid & 0.02296 \\
\hline Calcaneo-Cuboid Dorsal & Calcaneus & - & Cuboid & 0.02543 \\
\hline Talo-Navicular Dorsal & Talus & - & Navicular & 0.01164 \\
\hline Talo-Navicular Dorsal & Talus & - & Navicular & 0.01164 \\
\hline Calcaneo-Navicular Bifurcate & Calcaneus & - & Navicular & 0.01659 \\
\hline Calcaneo-Cuboid Bifurcate & Calcaneus & - & Cuboid & 0.01422 \\
\hline TarsoMetatarsal Dorsal & Medial Cuneiform & - & $1^{\text {st }}$ Metatarsal & 0.01271 \\
\hline TarsoMetatarsal Dorsal & Inter. Cuneiform & - & $2^{\text {nd }}$ Metatarsal & 0.00963 \\
\hline TarsoMetatarsal Dorsal & Lateral Cuneiform & - & $3^{\text {rd }}$ Metatarsal & 0.00684 \\
\hline TarsoMetatarsal Dorsal & Cuboid & - & $4^{\text {th }}$ Metatarsal & 0.00852 \\
\hline TarsoMetatarsal Dorsal & Cuboid & - & $5^{\text {th }}$ Metatarsal & 0.00733 \\
\hline TarsoMetatarsal Plantar & Medial Cuneiform & - & $1^{\text {st }}$ Metatarsal & 0.00903 \\
\hline TarsoMetatarsal Plantar & Inter. Cuneiform & - & $2^{\text {nd }}$ Metatarsal & 0.00745 \\
\hline TarsoMetatarsal Plantar & Lateral Cuneiform & - & $3^{\text {rd }}$ Metatarsal & 0.00697 \\
\hline TarsoMetatarsal Plantar & Cuboid & - & $4^{\text {th }}$ Metatarsal & 0.00767 \\
\hline TarsoMetatarsal Plantar & Cuboid & - & $5^{\text {th }}$ Metatarsal & 0.00897 \\
\hline
\end{tabular}




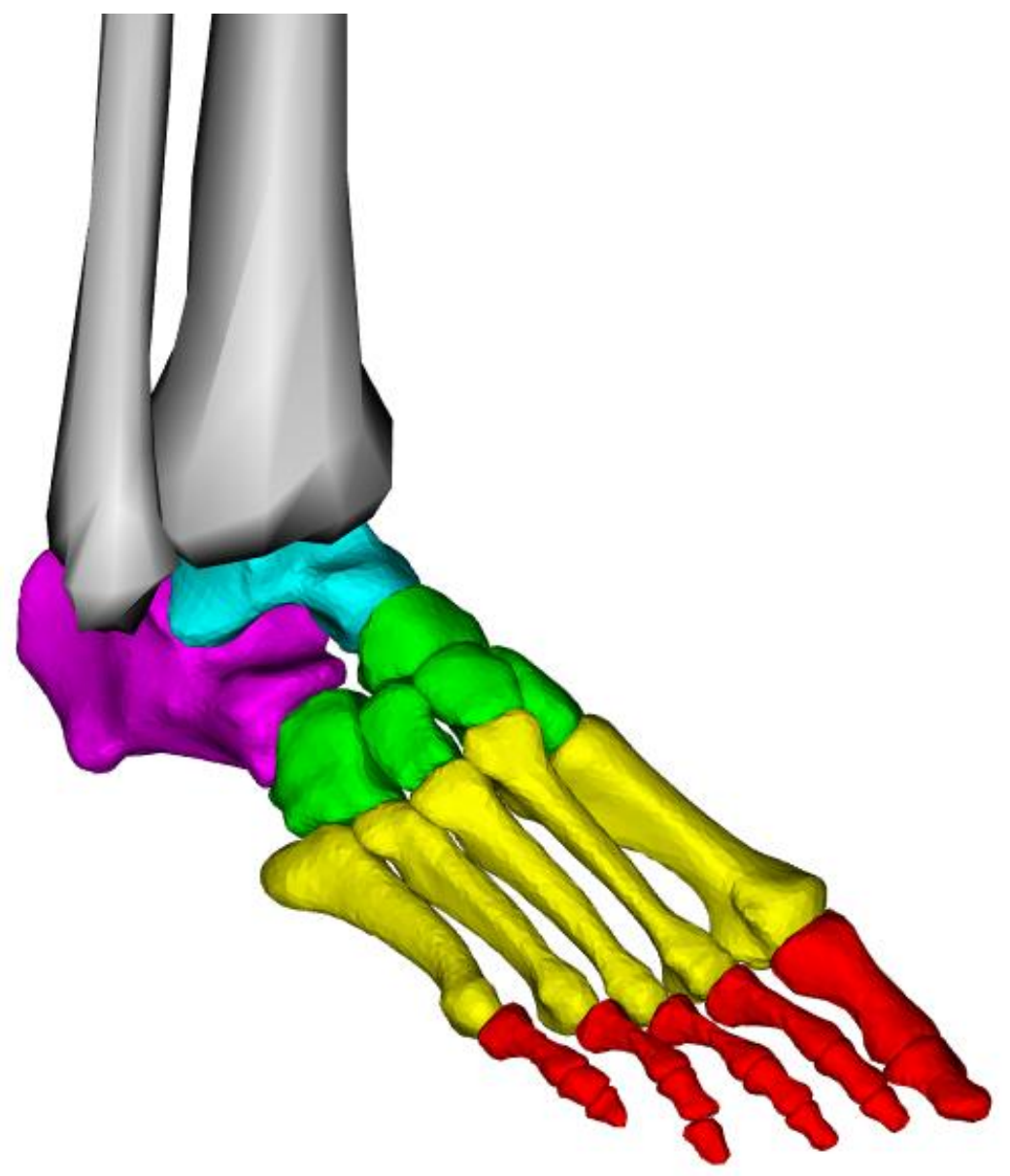

Figure 1 - Extended foot model (OpenSim). Depicted segments: Cyan - Talus; Purple Calcaneus; Green - Midfoot; Yellow - Forefoot; Red - Toes. 
a)


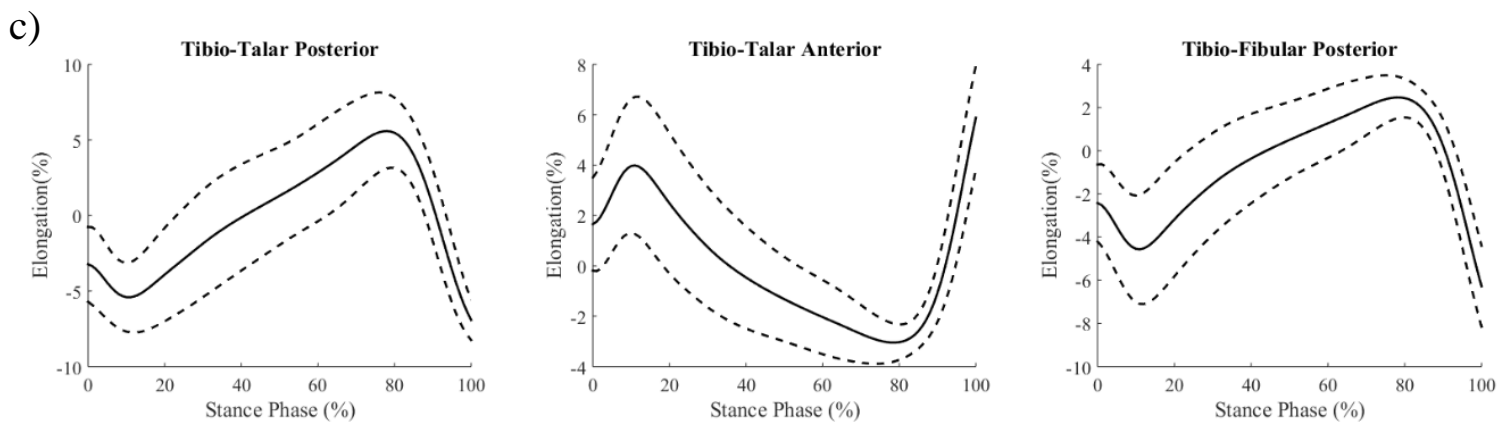

Figure 2 - Results: (a) Kinematic results - Angles (degrees) between the two specified bodies for the 8DOF model (green), 15DOF model (red) and Lundgren et al. (2008) (blue) during the stance phase of gait; (b) Inverse Dynamics results - Joint normalised moments ( $\mathrm{Nm} / \mathrm{Kg}$ ) obtained for the 8DOF model during the stance phase of gait; (c) Ligaments - Elongation of the right foot ligaments (\% of their resting length) during the stance phase of gait. The solid lines represent the mean of the trials and the dashed lines \pm SSD. 